\title{
Reisediarrhöen
}

\section{Vorbeugen ist schwieriger als heilen}

\author{
Auf einigen Nilkreuzfahrten sind bis zu 80\% der Passagiere betroffen, bei \\ Reisen nach Mittel- und Südamerika erleidet jeder Zweite eine Diarrhö. Damit \\ Ihre reiselustigen Patienten nicht auch Opfer von Pharaos oder Montezumas \\ Rache werden, sollten Sie sie über die Möglichkeiten einer Infektionsprophyla- \\ xe informieren. Und beim Reiserückkehrer mit Diarrhöen stellt sich die Frage, \\ ob und wenn ja welches Antibiotikum verordnet werden sollte.
}

— Jedes Jahr reisen etwa acht Millionen Deutsche in tropische und subtropische Regionen, sagte Prof. Rüdiger Braun, Esslingen. Nur wenige informieren sich vorher ausreichend über die vor Ort bestehenden Gefährdungen und die empfohlenen Hygienemaßnahmen.

\section{Diarrhöen am häufigsten}

Bei den auf Reisen erworbenen Infektionen stehen Diarrhöen mit Abstand an erster Stelle. „Magen-Darm-Infektionen werden insbesondere bei Aufenthalten in Afrika, weiten Teilen Asiens und Mittelamerikas erworben“, so Braun. „Jeder Zweite, der länger als zwei Wochen in solchen Regionen unterwegs ist, erkrankt an einer bakteriellen Diarrhö.“ Die häufigsten Erreger sind enteropathogene E. coli, gefolgt von Salmonellen, Shigellen und Campylobacter, wobei das Erregermuster von Region zu Region schwankt. „Die Kenntnis des Reiseziels ist für die Planung einer Prophylaxe und einer kalkulierten Therapie unabdingbar", so Braun.

\section{Vorrangig ist der Flüssigkeits- und Elektrolytersatz}

Wichtigste Therapie bei Reisediarrhö ist der Flüssigkeits- und Elektrolytersatz. Motilitätshemmer wie Loperamid sollten zurückhaltend eingesetzt werden, da hierdurch die Erreger im Darm länger verbleiben und somit die Diarrhöen länger anhalten.

Braun hält eine antibiotische Therapie nur bei schweren Verläufen mit klarer Keimassoziation erforderlich. Eingesetzt werden können Gyrasehemmer wie Ciprofloxacin, Cotrimoxazol und Makrolide. Als Therapie der Wahl gilt heute Ciprofloxacin. In Gebieten mit hoher ChinolonResistenz empfiehlt sich Cotrimoxazol.

\section{Medikamentöse Prophylaxe?}

Für die Prophylaxe von Reiseinfektionen gilt seit ewigen Zeiten: Peel it, cook it or forget it. Doch hieran halten sich leider nur wenig Reisende, sodass zusätzliche prophylaktische Maßnahmen einen immer größeren Stellenwert bekommen. Dies betrifft insbesondere Patienten mit Immunsuppression oder mit entzündlichen Darmerkrankungen, für die eine Darminfektion während der Reise eine erhebliche Gefährdung darstellt. Für die zur Prophylaxe häufig eingesetzten Probiotika konnte nach Braun bisher keine ausreichende Schutzwirkung nachgewiesen werden. Der generelle prophylaktische Einsatz von systemisch wirksamen Antibiotika biete eine ungünstige Nutzen-Risiko-Relation und

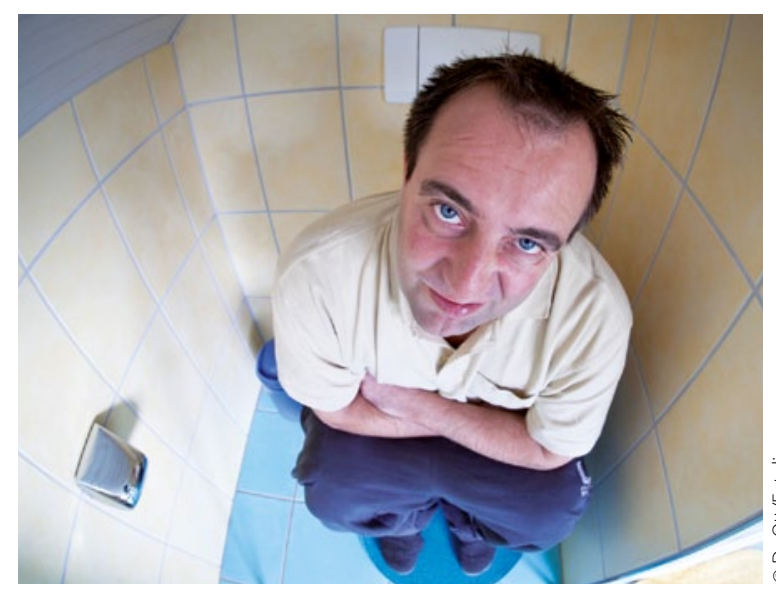

Nicht der Aufenthaltsort, von dem Urlauber träumen. beinhalte auch die Gefahr einer Resistenzbildung. „Nicht resorbierbare Antibiotika wie Rifaximin können zwar theoretisch verordnet werden, sind in dieser Indikation aber bisher in Deutschland nicht zugelassen", so Braun.

\section{Was gehört in die Reiseapotheke?}

Für die Mehrzahl der Reisenden gilt: Ein Antibiotikum sollte erst dann eingesetzt werden, wenn Symptome auftreten. „Es empfiehlt sich, Ciprofloxacin, Cotrimoxazol und Metronidazol (z. B. Clont ${ }^{\circ}$ ) mitzunehmen“, so Braun. Letzteres sei insbesondere bei Nilkreuzfahrten sinnvoll, wo häufig eine Amoebenruhr auftrete. Da die medizinische Betreuung in vielen Reiseländern nicht dem Standard in Deutschland entspreche, sollte der Patient die antibiotische Therapie bedarfsorientiert selbst beginnen.

\section{Erst essen, dann trinken!}

Dass die Magensäure ein wichtiger protektiver Faktor bei der Verhinderung einer Darminfektion ist, gilt als gesichert. „Durch Trinken wird die Magensäure verdünnt, was das Infektionsrisiko erhöhen kann", so Braun. Deshalb sei es gerade auf Reisen sinnvoll, zunächst zu essen und dann erst zu trinken. Da auch Protonenpumpenhemmer die Schutzwirkung des Magensaftes beeinträchtigen, sollten sie während der Reise abgesetzt werden. Ist dies nicht möglich, sollte evtl. eine antibiotische Prophylaxe erfolgen. Die wirksamste Prophylaxe neben den Allgemeinmaßnahmen sind Impfungen gegen Salmonellen (z. B. Thyphora $\mathrm{L}^{\bullet}$ ) oder gegen Cholera $\left(\right.$ Dukoral $\left.^{\circ}\right)$. Neue Impfstoffe haben die Immunität gegen Enterotoxine und die Adhärensfaktoren von E. coli in Form einer Schluckimpfung zum Ziel.

Dr. med. Peter Stiefelhagen -

- Quelle: XIV. Gastroenterologie-Seminarwoche, 28.2.2012 in Titisee 